\title{
The WFSA and patient safety in the perioperative setting
}

\author{
Angela Enright, MB · Alan Merry, MB
}

Published online: 20 December 2008

(c) Canadian Anesthesiologists' Society 2008

Since its foundation in 1955, the World Federation of Societies of Anaesthesiologists (WFSA) has dedicated its resources to improving the standard of anesthesia world wide. It does this in a variety of ways - through education programs, publications, scientific collaboration, and the setting of standards for anesthesia practice.

An independent International Task Force on Anaesthesia Safety developed a set of standards which were adopted by WFSA in $1992 .{ }^{1}$ These standards were updated and approved by the general assembly of WFSA in March of 2008. They are recommended for professionals throughout the world and are available on the WFSA website. ${ }^{2}$

In many areas of the world, these standards are already met or are even exceeded. However, in environments where resources are scarce, achieving these standards may remain a distant goal, yet a worthwhile goal nevertheless. In Uganda, a recent survey ${ }^{3}$ delineated the need, not only for monitors and machines, but also for some of the most basic essentials for safe anesthesia. Eighty percent of

Dr. A. Enright is the President of the World Federation of Societies of Anaesthesiologists (WFSA).

Dr. A. Merry is Chair of the Safety and Quality of Practice Committee of the WFSA.

A. Enright, MB

Department of Anesthesia, Royal Jubilee Hospital,

Victoria, BC, Canada

A. Merry, MB (ه)

Department of Anaesthesiology, University of Auckland,

Private Bag 92019, Auckland, New Zealand

e-mail: a.merry@auckland.ac.nz

A. Merry, MB

Department of Anaesthesia, Auckland City Hospital,

Auckland, New Zealand respondents did not always have electricity, 44\% lacked running water, and $30 \%$ did not always have intravenous fluids. Twenty-three percent of participants had the essentials to provide safe anesthesia to an adult, but only $6 \%$ could provide a safe anesthetic for Cesarean delivery. Only $13 \%$ of respondents could provide safe anesthesia to a child less than 5 years old. A similar survey in Moldova in August 2007 (G. Iohom, personal communication) demonstrated a lack of anesthesia equipment that many would consider essential to the provision of safe anesthesia. So how do WFSA standards apply in such settings?

In revising the standards, the WFSA has sought to identify those fundamental practices that, in the present day, should be the minimum observed during the provision of anesthesia for any elective procedure. Standards included in this basic category have been carefully limited to those likely to make a substantial difference to patient safety. Some may seem difficult to achieve, but all should be possible in any service providing elective surgery with general or major regional anesthesia.

As before, the most important safety monitor is the presence of a well-trained anesthesia professional. The WFSA views anesthesia as a medical practice. In many parts of the world, anesthesia is delivered by non-medical personnel. ${ }^{3}$ The WFSA believes that not only should these providers be formally trained and accredited, but they should also be supervised by medically qualified anesthesia specialists. Unfortunately, this is not always the case. In some places, anesthesia may be provided by practitioners with absolutely no training; furthermore, input from any medically qualified anesthesiologist is often absent (even in the minimal form of oversight from a distance). The WFSA believes this state of affairs to be unacceptable in 2008 .

Elevating the continuous use of a quantitative monitor of oxygenation, such as pulse oximetry, to the list of minimal 
requirements is an important advance on the earlier standards. This practice is already mandated by a large number of anesthesia societies and colleges in diverse countries, and the adoption of pulse oximetry as a standard of care is clearly one of the factors substantially contributing to improvements in the safety of anesthesia in the developed world over the last two decades. The time is past due that all patients undergoing anesthesia should benefit from this effective monitoring modality.

It is critically important to identify priorities when resources for health care are severely constrained. Should money be spent on primary health initiatives (such as vaccination programs) before it is spent on technology for surgery? If the answer is yes, then should the real question be whether elective surgery should be undertaken at all? In fact, surgery can be highly effective in relieving many causes of ill health and is an essential element of the response to the global burden of disease. Human suffering is relieved, to a great extent, by the 234 million operations undertaken worldwide each year. ${ }^{4}$ However, operations also cause harm, and avoidable complications of surgery have increasingly been recognized as an important public health problem. Even in developed countries, 3-16\% of hospitalized surgical patients develop major complications from their procedures, with permanent disability or death occurring in nearly $1 \% .^{5,6}$ In resource-limited settings, these figures are likely to be considerably higher, and much of the increased risk is attributable to anesthesia. In modern practice, anesthesia related mortality rates are considered to be very low. ${ }^{7}$ However, such is not the case in resource poor areas of the world. ${ }^{8}$ This is true for both surgical and obstetrical patients. In the report, published in 2006, concerning the confidential enquiry into maternal deaths in South Africa, ${ }^{9}$ it is noted that the vast majority of deaths that were classified as anesthetic related were thought to have been avoidable. The report recommends establishing guidelines for the equipment essential for provision of maternal care and advocates that skills in anesthesia be improved at all levels of health care. Some countries have reported alarming rates of anesthesia mortality (e.g., 1 in 1,900 patients anesthetized in Zambia ${ }^{10}$ and 1 in 150 patients anesthetized in Togo). ${ }^{11}$ In fact, at a certain level of risk, the provision of surgery is a disservice, contributing net harm to a community rather than net benefit. The reality is that surgery cannot be safely undertaken without at least some investment in staff and infrastructure. If assessed on a per patient basis, pulse oximetry is no longer novel or even particularly expensive. Its value in the early identification of hypoxia is proven, ${ }^{12}$ and there is compelling, albeit indirect, evidence to support its pivotal value in increasing patient safety. ${ }^{13}$ No one would seriously suggest that it would be acceptable for a Canadian surgical service to operate without pulse oximetry. Likewise, there is simply no justification for arguing that this practice would be acceptable elsewhere (it is acknowledged in the standards that it may be a different matter in the case of an emergency, where the life or limb of a patient may be at stake). On the contrary, there is every reason to insist that an oximeter, along with a sterilizer, is an essential requirement for safe surgery. If an oximeter is not available, elective surgery should be restricted to procedures achievable with local anesthesia.

The mere availability of pulse oximetry will not, in and of itself, improve patient care. Anesthesia providers must actually make use of these monitors, and respond appropriately to the information they provide. This implies certain other resources, such as oxygen and the means to manage hypoventilation and maintain a patent airway. It also implies a fairly comprehensive knowledge of the relevant physiology, and an understanding of clinical anesthesia. As stated above, there are anesthesia providers who do not have this knowledge. Pulse oximeters are needed, but so too is training. In fact, oximetry provides an excellent focus for teaching many of the basic principles of safe anesthesia. Furthermore, providing a package of oximetry and education can provide a powerful message to administrators responsible for running hospitals or accountable for the allocation of funding. The package can be used to emphasize that the provision of safe anesthesia is not trivial, and a reasonable investment in both equipment and expertise is essential. The Global Oximetry Project ${ }^{14}$ has accomplished just this, providing oximeters and education to anesthesiologists in four regions of the world. The World Health Organization has recognized the importance of anesthesia in the safe provision of surgery. ${ }^{15}$ Its Safe Surgery Saves Lives initiative has encapsulated pulse oximetry as an essential element on a safety checklist for use in all surgical procedures. ${ }^{16}$ As an extension to this initiative, a project to facilitate worldwide compliance with this requirement is now underway. The vision of every patient, everywhere, benefiting from oximetry during anesthesia may become a reality in the foreseeable future.

The WFSA is likely to play a major role in ensuring that these oximeters are used appropriately. Through the work of its education committee, it has a long track record of training anesthesiologists. While the focus is definitely on educating physicians about anesthesia, there also remains a significant commitment to assist all anesthesia providers. The WFSA provides enhanced training in anesthesia at 11 centres throughout the world. The training may be about anesthesia in general (e.g., in Bangkok, Thailand or ClujNapoca, Romania) or in subspecialty anesthesia (e.g., pediatric anesthesia in Vellore, India). Funded by the WFSA, young anesthesiologists from areas of need can spend up to a year in such centres. They subsequently return home, 
teach others, and improve the standard of anesthesia in their area. The retention rate of such WFSA trainees in their home countries has been very high. Of the 44 trainees educated in the Bangkok program since 1996 (from countries such as Mongolia, Laos, Myanmar, Cambodia, and Vietnam), only one individual has subsequently emigrated. The WFSA has heard time and time again that the return of these trained anesthesiologists has made a huge difference to anesthesia practice. This is attested to by local surgeons who have taken the time to write to express their appreciation (R. Jacob, personal communication). In addition, this training better prepares these anesthesiologists to train and to supervise non-medical anesthesia providers.

In the end, safe anesthesia practice in any region depends on the knowledge and skills of those who live and work there. Education is the catalyst by which local standards can be improved, and it serves as the vehicle through which local aspirations to the internationally accepted standards of health care expected in the developed world can be realized.

\section{La WFSA et la sécurité des patients dans le cadre périopératoire}

Depuis sa création en 1955, la World Federation of Societies of Anaesthesiologists (WFSA), c'est-à-dire la Fédération mondiale des Sociétés d'anesthésiologistes, a consacré ses ressources à l'amélioration des normes d'anesthésie partout dans le monde. La Fédération remplit cette mission de plusieurs façons : par des programmes d'éducation, des publications, des collaborations scientifiques, et l'établissement de normes pour la pratique de l'anesthésie.

Un Groupe de travail international indépendant sur la sécurité en anesthésie (International Task Force on Anaesthesia Safety) a élaboré un ensemble de normes qui ont été adoptées en 1992 par la WFSA. ${ }^{1}$ Ces normes ont été mises à jour et approuvées par l'assemblée générale de la WFSA en mars 2008. Elles sont destinées aux professionnels des quatre coins de la planète et sont à leur disposition sur le site Internet de la WFSA. ${ }^{2}$

Dans plusieurs régions du monde, ces normes sont déjà en vigueur ou même dépassées. Toutefois, dans des environnements où les ressources sont rares, le respect de ces normes peut être un objectif distant - tout en en valant la peine. En Ouganda, un sondage récent ${ }^{3}$ a révélé le manque non seulement de moniteurs et de machines, mais également de certains des éléments de base permettant une anesthésie sécuritaire. Quatre-vingts pour cent des répondants ont affirmé ne pas toujours avoir d'électricité, $44 \%$ n'avaient pas d'eau courante, et $30 \%$ n'avaient pas toujours des liquides intraveineux sous la main. Vingt-trois pour cent des participants au sondage disposaient du matériel essentiel pour pratiquer une anesthésie sécuritaire chez un adulte, mais seulement $6 \%$ étaient en mesure de prodiguer une anesthésie sécuritaire pour une césarienne. Seulement $13 \%$ des répondants pouvaient administrer une anesthésie sécuritaire à un enfant de moins de cinq ans. Une étude semblable menée en Moldavie au mois d'août 2007 (G. Iohom, communication personnelle) a montré un manque d'équipement anesthésique que nombre d'entre nous considèrent essentiel pour la prestation d'une anesthésie sécuritaire. Dès lors, comment les normes de la WFSA s'appliquent-elles dans de tels contextes?

Lors de la révision de ces normes, la WFSA s'est efforcée d'identifier les pratiques fondamentales qui, à l'heure actuelle, devraient constituer le minimum requis pour offrir une anesthésie dans n'importe quelle intervention non urgente. Lors de l'élaboration des normes comprises dans cette catégorie de base, nous avons fait attention à limiter notre liste à celles qui pourraient probablement faire une différence considérable dans la sécurité des patients. Certaines de ces normes peuvent paraître difficiles à atteindre, mais l'ensemble devrait être réalisable dans n'importe quel service offrant des services de chirurgie non urgente avec une anesthésie générale ou régionale majeure.

Tout comme par le passé, les nouvelles normes réaffirment une fois de plus que le moniteur le plus important pour la sécurité demeure la présence d'un professionnel de l'anesthésie bien formé. La WFSA considère l'anesthésie comme une pratique médicale. Dans plusieurs régions du monde, l'anesthésie est prodiguée par du personnel non médical. ${ }^{3}$ Selon la WFSA, ces prestataires d'anesthésie devraient non seulement être formés et certifiés de façon formelle, mais ils devraient également être supervisés par des spécialistes de l'anesthésie qualifiés d'un point de vue médical. Malheureusement, ce n'est pas toujours le cas. Dans certains endroits, l'anesthésie peut être administrée par des praticiens n'ayant absolument aucune formation; de plus, les contributions et conseils d'un anesthésiologiste qualifié au niveau médical sont souvent inexistants (il n'y a souvent même pas de supervision à distance). La WFSA estime cet état des choses fondamentalement inacceptable en 2008.

L'ajout de l'utilisation continue d'un moniteur quantitatif de l'oxygénation, comme l'oxymétrie de pouls, à notre liste des exigences minimales est un progrès significatif comparativement aux normes précédentes. Cette pratique est déjà recommandée par nombre de sociétés et de collèges d'anesthésie dans plusieurs pays, et l'adoption de l'oxymétrie de pouls en tant que norme de soins constitue 
clairement l'un des facteurs qui contribue de façon importante aux améliorations apportées à la sécurité de l'anesthésie dans le monde développé depuis ces vingt dernières années. Il est grand temps que tous les patients subissant une anesthésie profitent de cette modalité de monitorage efficace.

L'identification des priorités est d'une importance cruciale lorsque les ressources pour les soins de santé sont très limitées. L'argent devrait-il aller aux initiatives de soins de santé primaires (comme par exemple des programmes de vaccination) avant d'être dépensé pour les technologies nécessaires à la chirurgie ? Si nous répondons par l'affirmative, la véritable question ne serait-elle pas dès lors de décider si la chirurgie non urgente doit être ou non réalisée ? En fait, la chirurgie peut être très efficace pour soulager de nombreuses causes de mauvaise santé et constitue un élément essentiel du combat contre la maladie dans le monde. La souffrance humaine est soulagée, en grande partie, par les 234 millions d'opérations réalisées chaque année dans le monde. ${ }^{4}$ Toutefois, les opérations peuvent également faire du tort, et les complications chirurgicales qui auraient pu être évitées sont de plus en plus considérées comme un problème important de santé publique. En effet, et ce même dans les pays développés, $3-16 \%$ des patients chirurgicaux hospitalisés souffrent de complications majeures à la suite de l'intervention qu'ils ont subie, et presque $1 \%$ de ces patients souffrent de handicap permanent ou décèdent de ces complications. ${ }^{5,6}$ Dans les environnements où les ressources sont limitées, ces chiffres sont probablement considérablement plus élevés, et une grande partie de ces risques accrus est attribuable à l'anesthésie. Dans la pratique moderne, les taux de mortalité associés à l'anesthésie sont considérés très bas. ${ }^{7}$ Toutefois, ce n'est pas le cas dans les régions du monde où les ressources manquent, ${ }^{8}$ et ceci est valable en chirurgie aussi bien qu'en obstétrique. Dans le rapport publié en 2006 au sujet d'une enquête confidentielle concernant les décès maternels en Afrique du Sud, ${ }^{9}$ les auteurs ont observé que la vaste majorité des décès considérés comme associés à l'anesthésie auraient probablement pu être évités. Le rapport recommande que des directives soient établies en ce qui concerne le matériel essentiel à la prestation de soins à la mère et préconise que les compétences en anesthésie soient améliorées à tous les niveaux des soins de santé. Certains pays ont fait état de taux alarmants de décès dus à l'anesthésie (par ex., un patient sur 1900 anesthésié en Zambie ${ }^{10}$ et un sur 150 au Togo). ${ }^{11}$ En fait, lorsqu'on atteint un certain niveau de risque, la prestation chirurgicale fait du tort et apporte un mal net plutôt qu'un bienfait net à la communauté. En réalité, une chirurgie ne peut pas être réalisée de façon sécuritaire sans qu'il y ait au moins un certain investissement au niveau du personnel soignant et des ressources. Si elle est évaluée sur une base par patient, l'oxymétrie de pouls n'est plus ni nouvelle ni particulièrement chère. Sa contribution à l'identification précoce de l'hypoxie est prouvée, ${ }^{12}$ et il existe des données probantes convaincantes - quoique indirectes - qui lui accordent un rôle crucial pour augmenter la sécurité des patients. ${ }^{13}$ Il est impensable de suggérer qu'il soit acceptable pour un service chirurgical canadien d'opérer sans oxymétrie du pouls. De la même façon, il n'existe tout bonnement pas de justification pour soutenir cette pratique ailleurs dans le monde. Les normes font la place aux cas d'urgence, dans lesquels il est reconnu que la situation puisse être différente, si la vie ou un membre d'un patient est en jeu. En revanche, il est tout à fait justifié d'insister qu'un oxymètre, tout comme un stérilisateur, constituent des exigences essentielles à une chirurgie sécuritaire. Si il n'y a pas d'oxymètre disponible, les chirurgies non urgentes devraient se limiter à des interventions réalisables sous anesthésie locale.

Le simple fait de rendre disponible l'oxymétrie du pouls n'améliorera toutefois pas en soi les soins aux patients. Les personnes fournissant l'anesthésie doivent en fait utiliser ces appareils de monitorage et réagir de façon adéquate aux informations que ces appareils fournissent. Ceci implique dès lors d'autres ressources, comme de l'oxygène et des moyens de prendre en charge une hypoventilation ainsi que de maintenir les voies aériennes d'un patient. Mais cela implique également une connaissance relativement complète de la physiologie en question, ainsi qu'une compréhension de l'anesthésie clinique. Comme nous l'avons déjà mentionné plus haut, certaines personnes prodiguant des soins anesthésiques ne possèdent pas ces connaissances. Les oxymètres de pouls sont nécessaires, assurément, mais la formation est tout aussi importante. En fait, l'oxymétrie est un excellent moyen d'enseigner plusieurs des principes de base d'une anesthésie sécuritaire. En outre, la prestation d'une 'mallette pédagogique' offrant à la fois oxymétrie et formation pourrait transmettre un message fort aux administrateurs en charge de la gestion des hôpitaux ou responsables de l'attribution des fonds. Cette mallette peut servir à souligner que la prestation d'une anesthésie sécuritaire n'est pas à prendre à la légère, et qu'un investissement raisonnable dans les équipements et l'expertise est essentiel. Le Projet d'oxymétrie mondial (Global Oximetry Project) $^{14}$ a atteint précisément cet objectif en fournissant des oxymètres et une formation à des anesthésiologistes dans quatre régions du monde. L'Organisation mondiale de la Santé (OMS) a reconnu l'importance de l'anesthésie dans la prestation sécuritaire de chirurgie. ${ }^{15}$ L'initiative « Safe Surgery Saves Lives » (« La chirurgie sécuritaire sauve des vies ») de l'OMS a incorporé l'oxymétrie du pouls en tant qu'élément essentiel d'une liste de contrôle de sécurité à être utilisée dans toutes les interventions chirurgicales. ${ }^{16}$ Dans la continuité de cette 
initiative, un projet est actuellement en cours pour faciliter le respect de cette exigence à l'échelle mondiale. Que chaque patient, où qu'il soit soigné, profite de l'oxymétrie pendant l'anesthésie : voici une vision qui pourrait devenir réalité dans un proche avenir.

Il est probable que la WFSA joue un rôle majeur pour s'assurer que ces oxymètres soient utilisés de façon adéquate. Grâce au travail de son comité d'éducation, la Fédération possède une longue histoire en matière de formation des anesthésiologistes. Bien que l'objectif soit évidemment d'éduquer les médecins en anesthésie, notre engagement vise aussi à assister tous les prestataires d'anesthésie. La WFSA offre une formation améliorée en anesthésie dans 11 centres répartis dans le monde. La formation peut porter sur l'anesthésie en général (par ex. à Bangkok, en Thailande, ou à Cluj-Napoca, en Roumanie) ou sur une sous-spécialité de l'anesthésie (par ex. l'anesthésie pédiatrique à Vellore, en Inde). Grâce au financement de la WFSA, de jeunes anesthésistes provenant de régions démunies peuvent passer jusqu'à un an dans de tels centres. Ils retournent ensuite chez eux, enseignent à d'autres, et améliorent ainsi les normes de l'anesthésie dans leur région. Le taux de rétention des connaissances de tels stagiaires de la WFSA dans leur pays d'origine est très élevé. Parmi les 44 stagiaires éduqués dans le cadre du programme de Bangkok depuis 1996 (provenant de pays tels que la Mongolie, le Laos, Myanmar, le Cambodge et le Vietnam), un seul a émigré par la suite. La WFSA ne cesse d'avoir des échos selon lesquels le retour de ces anesthésiologistes formés a fait une différence énorme dans la pratique de l'anesthésie. Ce fait est attesté par les chirurgiens locaux qui nous ont écrit pour exprimer leur appréciation (R. Jacob, communication personnelle). De plus, cette formation prépare mieux ces anesthésiologistes à former et superviser des prestataires d'anesthésie sans formation médicale.

En fin de compte, une pratique sécuritaire de l'anesthésie, dans quelque région que ce soit, dépend des connaissances et compétences des personnes qui vivent et travaillent dans cette région. L'éducation est le catalyseur grâce auquel les normes locales peuvent être améliorées, et elle est également le moyen par lequel les aspirations locales à des normes mondialement acceptées de soins de santé attendus dans le monde développé peuvent se concrétiser.

\section{Remerciements}

Les Normes internationales pour une pratique sécuritaire de l'anesthésie (International Standards for A Safe Practice of Anesthesia) ont été révisées par (par ordre alphabétique) : Meena Cherian, Jeff Cooper, John Eichhorn, Alan Merry,
Olaitan Soyanwo, et Iain Wilson. Le Projet mondial d'oxymétrie (Global Oximetry Project) a impliqué (entre autres, à nouveau par ordre alphabétique): Alan Merry, Florian Nuevo, Gavin Thoms, David Whitaker et Iain Wilson, et a été soutenu par General Electric Healthcare et l'Association of Anaesthetists of Great Britain and Ireland, ainsi que par la WFSA. L'initiative « Safe Surgery Saves Lives » a été menée par Atul Gawande. Nous tenons à remercier le Dr John H. Eichhorn pour avoir pris le temps de relire ce manuscrit et de le commenter.

Acknowledgements The International Standards for A Safe Practice of Anesthesia were revised by (in alphabetical order) Meena Cherian, Jeff Cooper, John Eichhorn, Alan Merry, Olaitan Soyanwo, and Iain Wilson. The Global Oximetry Project involved (among others, again in alphabetical order) Alan Merry, Florian Nuevo, Gavin Thoms, David Whitaker and Iain Wilson, and was supported by General Electric Healthcare and the Association of Anaesthetists of Great Britain and Ireland, as well as the WFSA. The Safe Surgery Saves Lives initiative was led by Atul Gawande. We thank Dr. John $\mathrm{H}$. Eichhorn for reading and commenting on the manuscript.

Conflict of interest Dr. Merry is a Board Member of Safer Sleep LLC.

\section{References}

1. The International Taskforce on Anaesthesia Safety. International standards for a safe practice of anaesthesia. Eur J Anaesthesiol 1993; 10: 12-5.

2. 2008 International Standards for a Safe Practice of Anaesthesia. www.anaesthesiologists.org. Accessed 20 Sept 2008.

3. Hodges SC, Mijumbi C, Okello M, McCormick BA, Walker IA, Wilson IH. Anaesthesia services in developing countries: defining the problems. Anaesthesia 2007; 62: 4-11.

4. Weiser TG, Regenbogen SE, Thompson KD, et al. An estimation of the global volume of surgery: a modelling strategy based on available data. Lancet 2008; 372: 139-44.

5. Gawande AA, Thomas EJ, Zinner MJ, Brennan TA. The incidence and nature of surgical adverse events in Colorado and Utah in 1992. Surgery 1999; 126: 66-75.

6. Kable AK, Gibberd RW, Spigelman AD. Adverse events in surgical patients in Australia. Int J Qual Health Care 2002; 14: 26976.

7. Gibbs N, Borton C. Safety of Anesthesia in Australia. A review of Anaesthesia Mortality 2000-2002. Melbourne: Australian and New Zealand College of Anaesthetists; 2006.

8. Walker I, Wilson I, Bogod D. Anaesthesia in developing countries. Anaesthesia 2007; 62: 2-3.

9. National Committee on Confidential Enquiries into Maternal Deaths. Saving mothers - report of confidential enquiry in maternal deaths in South Africa 2002-2004. http://www.doh.gov. za/docs/reports/2004/savings.pdf. Accessed 20 Sept 2008.

10. Heywood AJ, Wilson IH, Sinclair JR. Perioperative mortality in Zambia. Ann R Coll Surg Engl 1989; 71: 354-8.

11. Ouro-Bang'na Maman AF, Tomta K, Ahouangbevi S, Chobli M. Deaths associated with anaesthesia in Togo, West Africa. Trop Doc 2005; 35: 220-2.

12. Moller JT, Johannessen NW, Espersen $K$, et al. Randomized evaluation of pulse oximetry in 20,802 patients: II. Perioperative 
events and postoperative complications. Anesthesiology 1993; 78: 445-53.

13. Runciman WB. Iatrogenic harm and anaesthesia in Australia. Anaesth Intensive Care 2005; 33: 297-300.

14. Thoms GM, McHugh GA, O'Sullivan E. The Global Oximetry initiative. Anaesthesia 2007; 62: 75-7.
15. Cherian MN, Merry AF, Wilson IH. The World Health Organization and anaesthesia. Anaesthesia 2007; 62: 65-6.

16. The WHO Surgical Safety Checklist First Edition. www.who.int/ patientsafety/challenge/safe.surgery/en/index.html. Accessed 27 Sept 2008. 